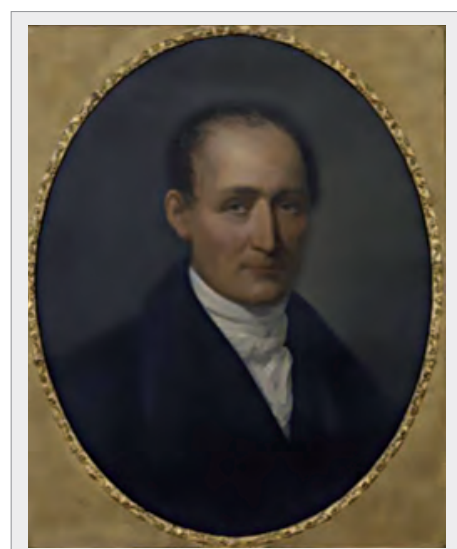

Portrait par Léonard-François Berger

\section{Principales dates}

7 mars 1792 - Naissance à Slough

(Buckinghamshire, Angleterre)

1825

Médaille Lalande de l'Académie

des Sciences de Paris

1830 Discourse on Natural Philosophy

1833 Royal Medal de la Royal Society

11 mai 1871 - Décès à Hawkhurst (Kent, Angleterre)

\title{
Joseph Nicéphore Niépce
}

\section{Riad Haidar}

haidar@onera.fr

Physicien français d'une grande ingéniosité, pionnier du moteur à explosion, inventeur original et fécond, Joseph Nicéphore Niépce est universellement connu aujourd'hui pour l'invention de la photographie.

$\mathrm{J}$ oseph Niépce naît le 7 mars 1765 à Chalon-sur-Saône. Il est le cadet d'une famille aisée de la bourgeoisie bourguignonne. Son père Claude, avocat de formation, gère les vastes propriétés de la famille et est conseiller du Roi ; sa mère Claudine, née Barault, est la fille d'un célèbre avocat.

Joseph reçoit une éducation classique, qu'il complète en sciences et en méthodologie expérimentale au collège des Oratoriens de Chalon-sur-Saône à partir de 1780, et plus tard à Angers. II se destine à la prêtrise et adopte en 1787 le prénom de Nicéphore (le «porteur de victoire »), en hommage à Nicéphore ler, patriarche de Constantinople au IXe siècle. Ses excellents résultats scolaires lui permettent d'intégrer le corps professoral de son collège à Angers, puis à Troyes. Mais, à la veille de la Révolution, il renonce à la prêtrise. II vient d'avoir 24 ans, et milite activement pour l'abolition de l'ordre ancien. II rejoint la Garde Nationale en 1792 , et participe à des campagnes dans le sud de la France et en Sardaigne. En 1794, des soucis de santé coupent court à sa carrière militaire. II s'établit alors à Nice et épouse la fille de sa logeuse, Agnès Roméro. Ils auront un fils, Isidore, né en 1795.

À Nice, où le rejoint son frère aîné Claude, Niépce occupe le poste d'administrateur de district (l'équivalent d'un arrondissement sous la Révolution). Mais, peutêtre à cause $d^{\prime}$ une certaine impopularité auprès de la population locale, il démissionne de sa charge en 1795. II décide en 1798, avec son frère Claude, de se consacrer à une carrière $d$ 'inventeur. Ingénieux, férus de physique et de chimie, les deux frères débordent en effet de créativité scientifique et de talent pragmatique.

\section{Premiers travaux}

En 1801, tous regagnent la propriété des Niépce à Saint-Loup-de-Varennes, à quel ques kilomètres de Chalon-sur-Saône.

\section{Micro positionnement pour l'usinage laser}

Les contrôleurs, les platines et les systèmes Aerotech augmentent la précision et le rendement de votre application tout en
maximisant les temps de bon fonctionnement. PRO Series

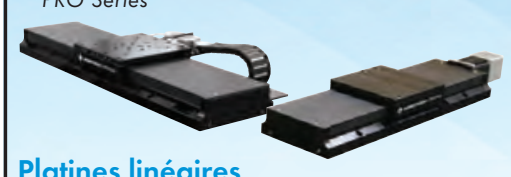

Platines linéaires

- Courses de 50 à $1500 \mathrm{~mm}$

- Vitesse jusqu'à $2 \mathrm{~m} / \mathrm{s}$

- Conçus avec des joints latéraux et un capot de protection en tôle

- Entrainement à vis à bille ou à moteur linéaire

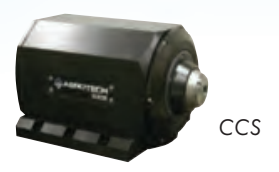

Rotation à entrainement direct

- Mandrin à serrage et desserrage pneumatique

- Collet captif minimise les défauts axiaux pendant le serrage/desserrage

- Ouverture central pour alimentation

- Moteur brushless et codeur dans I'axe

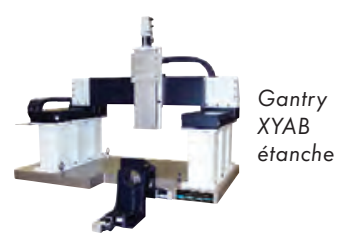

Systèmes Gantry

à moteurs linéaires

- Vitesse de $3 \mathrm{~m} / \mathrm{s}$ et accélération de $5 \mathrm{~g}$

- Précision exceptionnelle, haut rendement et grande productivité

- Versions étanches et options customisées pour votre application

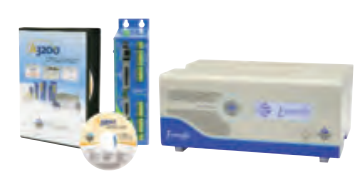

Plateformes contrôleurs avancés

- Mono et multiaxes

- Fonctions de contrôle avancées et software modulaire

- Environnement d'automation embarqué PLC

Télécharger notre brochure

Télécharger notre brochure
de solution pour process laser sur www.aerotech.com

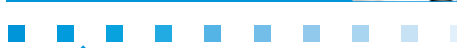
Dedicated to the Science of Motion Aerotech France, BP 70043 45702 Villemandeur Cedex Ph: +33 238970830 Email:ventes@aerotech.com

www.aerotech.com 
À trente-six ans, Niépce retrouve donc sa terre natale, et toute sa famille. Les années suivantes sont consacrées à la gestion du patrimoine familial, dont la mère s'occupait depuis le décès du père en 1785 et, surtout, à ses inventions scientifiques. Avec Claude, ils mettent au point le pyréolophore. Ce moteur, le premier à combustion interne de l'Histoire, parvient à propulser une maquette de bateau longue de 2 mètres sur la Saône. En 1807, les frères obtiennent un brevet valable dix ans mais, malgré l'appui de Lazare Carnot, ils ne parviennent pas à exploiter leur invention en France. Leur ingéniosité trouve d'autres exutoires, comme par exemple en 1809, la mise au point d'une pompe hydraulique pour remplacer la machine de Marly, devenue obsolète, qui assure l'alimentation en eau des châteaux de Marly et de Versailles; mais ils se manifestent trop tard auprès des autorités et leur dispositif n'est pas retenu...

Au final, aucun de leur projet n'a de succès commercial. Or l'état de guerre permanent et leurs coûteux travaux de recherche grèvent lourdement les finances de la famille. Les Niépce contractent en 1814 le premier d'une longue série d'emprunts. En 1816, à l'approche de l'expiration du brevet sur le pyréolophore, Claude décide de gagner l'Angleterre et d'y poursuivre, seul, l'aventure sur le « mouvement perpétuel».

\section{L'invention de la photographie}

Depuis plusieurs années, Nicéphore se passionne pour la lithographie, mise au point en 1796 par l'acteur et dramaturge autrichien Aloys Senefelder [1771-1834]. II s'en inspire pour tenter de fixer les images projetées au fond des chambres obscures (les fameuses camera obscura utilisées par les peintres). C'est un vieux rêve que d'autres ont partagé, tel notamment Jacques Charles [1746-1823] qui est parvenu, 30 ans plus tôt, à fixer, mais de façon éphémère, une silhouette sur du papier enduit de chlorure d'argent. Plus récemment, Thomas Wedgwood [17711805] a proposé en 1802 une méthode à base de nitrate d'argent, qui donne des images tout aussi fugitives.
En 1816, après le départ de son frère, Nicéphore fait ses premières tentatives. Comme ses prédécesseurs, il utilise le chlorure $d^{\prime}$ 'argent, mais sans plus de succès. C'est frustrant: $d$ 'abord le miracle semble s'opérer, les images se détachent nettement sur le papier, puis elles s'estompent très vite à la lumière... II persévère, et multiplie les essais pendant les années qui suivent. En 1822, enfin, il obtient des résultats plus pérennes avec le bitume de Judée, une substance noire qui blanchit et, surtout, durcit à la lumière : il réussit ses premières images en relief, qui confirment la pertinence de son procédé. Les succès s'enchaînent. En 1824, il réalise ses points de vue à la chambre obscure sur des pierres lithographiques, avec un temps de pose de 5 jours. En 1826, il grave des images sur du cuivre par la méthode des eaux-fortes (c'est de l'acide nitrique). Nicéphore peut enfin écrire à son frère : « la réussite est complète ». Il obtient, par cette technique qu'il appelle héliographie, après une dizaine d'heures d'exposition, ce qui constitue la toute première photographie : une vue prise d'une fenêtre de sa maison de Saint-Loup-de-Varennes.

L'année 1827 est une année charnière. La situation financière des Niépce est désormais exsangue, et la famille songe à vendre des propriétés pour rembourser des créanciers devenus impatients. Par ailleurs Claude, toujours en Angleterre, épuisé par ses recherches et l'échec de ses démarches pour négocier le pyréolophore, tombe gravement malade et sombre dans la démence. Nicéphore doit se rendre à son chevet.

Pourtant, malgré la tourmente, Niépce est conscient de la maturité de son invention. Il croit en son avenir commercial, et c'est pour lui un avenir à court terme. Pour perfectionner sa chambre obscure lqui est déjà dotée de quelques raffinements, tels que le diaphragme à iris ou la bobine pour l'enroulement du papier sensible), il $s$ 'adresse à des ingénieurs-opticiens établis à Paris, Vincent et Charles Chevalier, qui le conseillent et lui fournissent les optiques nécessaires. Surtout, Vincent Chevalier [1770-1841] le met en relation avec Louis Jacques Mandé Daguerre [1787-1851], un peintre décorateur, habile en affaires, qui utilise la chambre noire pour faire les
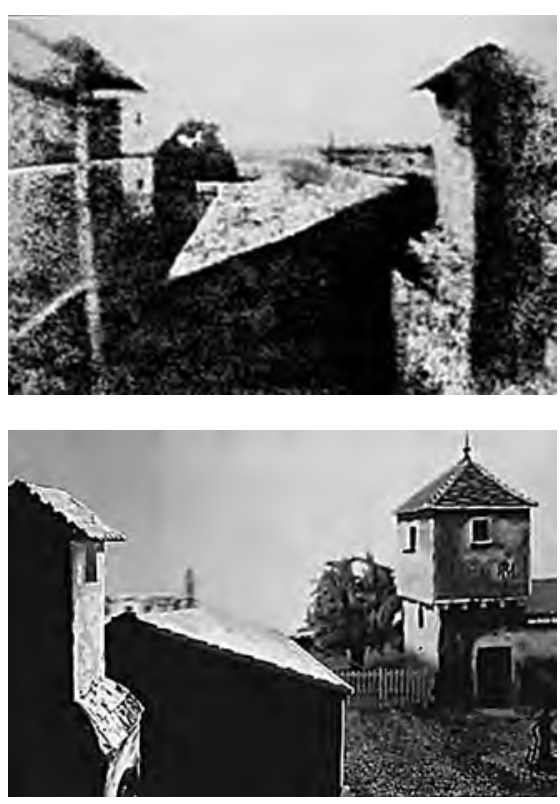

En haut, la photographie prise par Niépce de la vue de droite.

croquis de ses dioramas. L'inventeur chalonnais et le peintre parisien s'associent en octobre 1829, et entament une collaboration étroite. Ils travaillent sur toutes sortes de résines sans obtenir de résultats probants. En 1832, enfin, ils obtiennent des images avec un temps de pose de moins de 8 heures en utilisant comme produit photosensible le résidu de la distillation de l'essence de lavande. Ils appellent ce procédé prometteur physautotype. De toute évidence, ils sont sur la bonne voie...

Mais le soir du 5 juillet 1833, Nicéphore Niépce meurt subitement, probablement d'une hémorragie cérébrale, dans son domaine de Saint-Loup-de-Varennes - sans avoir pu mener l'aventure photographique à son terme. C'est à Daguerre qu'il revient $d$ 'apporter les perfectionnements nécessaires (notamment, la réduction du temps de pose et l'obtention d'une image définitive) pour rendre l'invention apte à une utilisation commerciale. Le daguerréotype, mis au point en 1838, lui apportera une grande célébrité et manquera même d'occulter l'apport séminal de Niépce.

\section{Référence}

[1] Jean-Louis Marignier, Niépce, l'invention de la photographie (Belin Paris, 1999).

[2] Mary Warner Marien, Photography: A Cultural History (Pearson, 2010). 